\title{
Management of endophthalmitis with pars plana vitrectomy
}

\author{
GHOLAM A. PEYMAN, ${ }^{1}$ MOTILAL RAICHAND, ${ }^{2}$ AND \\ THOMAS O. BENNETT ${ }^{1}$ \\ From the ${ }^{1}$ Department of Ophthalmology, University of Illinois Eye and Ear Infirmary, and \\ ${ }^{2}$ Cook County Hospital
}

SUMMARY Pars plana vitrectomy with intravitreal antibiotics is an effective technique in the treatment of culture-proved endophthalmitis. Best results are obtained when the therapy is instituted early in the course of infection, preferably during the first 36 hours of the onset of symptoms, and when the infective organism is of low virulence. In our 20 cases of culture-proved endophthalmitis 17 eyes $(85 \%)$ were saved and had some degree of useful vision. Thirteen $(65 \%)$ eyes had a visual acuity of $20 / 400$ or better. Three eyes $(15 \%)$ were lost.

Management of culture-proved endophthalmitis has been the subject of considerable therapeutic innovation. Laboratory and clinical studies have established intravitreal injection of antibiotics as an effective method of treating endophthalmitis. ${ }^{2}$ Beyond this form of treatment surgical intervention by pars plana vitrectomy has added another dimension to the treatment of endophthalmitis. ${ }^{13-5}$ This report describes our clinical experience in the management of culture-proved endophthalmitis with vitrectomy during a period of 5 years.

\section{Materials and methods}

Twenty cases of culture-proved endophthalmitis underwent vitrectomy by the Vitreous Service of the University of Illinois Eye and Ear Infirmary between 1974 and 1978. The clinical diagnosis of endophthalmitis was based on a combination of pain, periorbital oedema, conjunctival hyperaemia, chemosis, hypopyon, poor red reflex, or focal preretinal abscess. Ultrasonography and electroretinography aided in the evaluation. In cases of ocular trauma radiographs were obtained to rule out the presence of intraocular foreign bodies.

Anterior chamber and vitreous tapping were performed on all suspected cases of endophthalmitis. The samples of aqueous and vitreous were immediately inoculated on blood agar and chocolate agar plates, as well as on Sabouraud's media

Correspondence to Dr G. A. Peyman, University of Illinois Eye and Ear Infirmary, 1855 West Taylor Street, Chicago, Illinois 60612 , USA. for fungi and thioglycolate for anaerobes. Giemsa and Gram stains were also prepared on each specimen. After vitreous tap, a single injection of $400 \mu \mathrm{g}$ of gentamicin and $360 \mu \mathrm{g}$ of dexamethasone was administered into the anterior vitreous. All patients received systemic (intravenous) gentamicin for 3 days and topical antibiotics as needed.

Vitrectomy was scheduled 24 hours after intravitreal injection of antibiotics if the culture was positive. Immediate vitrectomy was performed in 2 clinical situations: (1) in bacterial endophthalmitis with extensive formation of vitreous abscess, and (2) in suspected cases of fungal endophthalmitis in which vitreous involvement was widespread and there was a delay in obtaining culture results. In both instances the deciding factor was the extent to which the vitreous clarity was involved. In such cases intravitreal antibiotics were not injected except as part of the vitrectomy surgery procedure. Surgery was performed with the patient under local anaesthesia except in apprehensive individuals. The vitrophage was used in all 20 cases. When the media were very cloudy, intraocular fiberoptic illumination was obtained with either a Fiberoptic sleeve built over the wide-angle cutter vitrophage or a separate light pipe. The technique of vitrectomy has been reported previously. ${ }^{1}$ Vitrectomy influsion fluid routinely contained $8 \mu \mathrm{g} / \mathrm{ml}$ of gentamicin.

When the culture results were known, appropriate antibiotics were added to the vitrectomy infusion fluid. especially if the organism was resistant to gentamicin. When the infecting organisms were not isolated or when mixed infection was suspected, a combination of antibiotics was added 
to the vitrectomy infusion fluid. ${ }^{67}$ In cases of fungal endophthalmitis an intravitreal injection of $5 \mu \mathrm{g}$ of amphotericin B was given after completion of vitrectomy. ${ }^{8}{ }^{9}$ In appropriate cases systemic and topical antibiotic therapy was initiated.

\section{Results}

In our series eight culture-proved endophthalmitis cases $(40 \%)$ (Table 1) occurred after previous surgical procedures. Trauma was involved in 8

Table 1 Conditions predisposing to endophthalmitis

\begin{tabular}{llr}
\hline Condition & No. of patients & $\%$ \\
\hline Cataract extraction & 5 & 20 \\
Phacoemulsification & 2 & 10 \\
Vitrectomy & 1 & 5 \\
Trauma & 8 & 40 \\
Following intravenous & 4 & 20 \\
$\quad$ heroin injection & & \\
\hline
\end{tabular}

additional patients $(40 \%)$. Four cases $(20 \%)$ developed candida endophthalmitis after intravenous drug abuse. The most common strains of organisms causing infection were: Staphylococcal (6 cases), streptococcal (4 cases), and candida organisms (5 cases) (Table 2). Aqueous cultures yielded positive results in only $15 \%$ of the specimens collected, while vitreous cultures proved positive in all cases. In 3 cases the eye was lost; 2 were infected with streptococcal organisms and one with Serratia marcescens.

From 14 cases of bacterial endophthalmitis only 1 (case 15) received a combination of antibiotics in the vitrectomy influsion fluid (gentamicin $8 \mu \mathrm{g} / \mathrm{ml}$ and clindamycin $10 \mu \mathrm{g} / \mathrm{ml}$ ). In none of the cases did we reinject antibiotics intravitreally after vitrectomy was performed. The eyes were generally inflamed for about a week after the surgery and gradually recovered.

$85 \%$ of the patients retained their eyes. $50 \%$ of eyes had a visual acuity equal to or better than $20 / 100$, and another $15 \%$ had a visual acuity between

Table 2 Summary of patients treated with vitrectomy for endophthalmitis

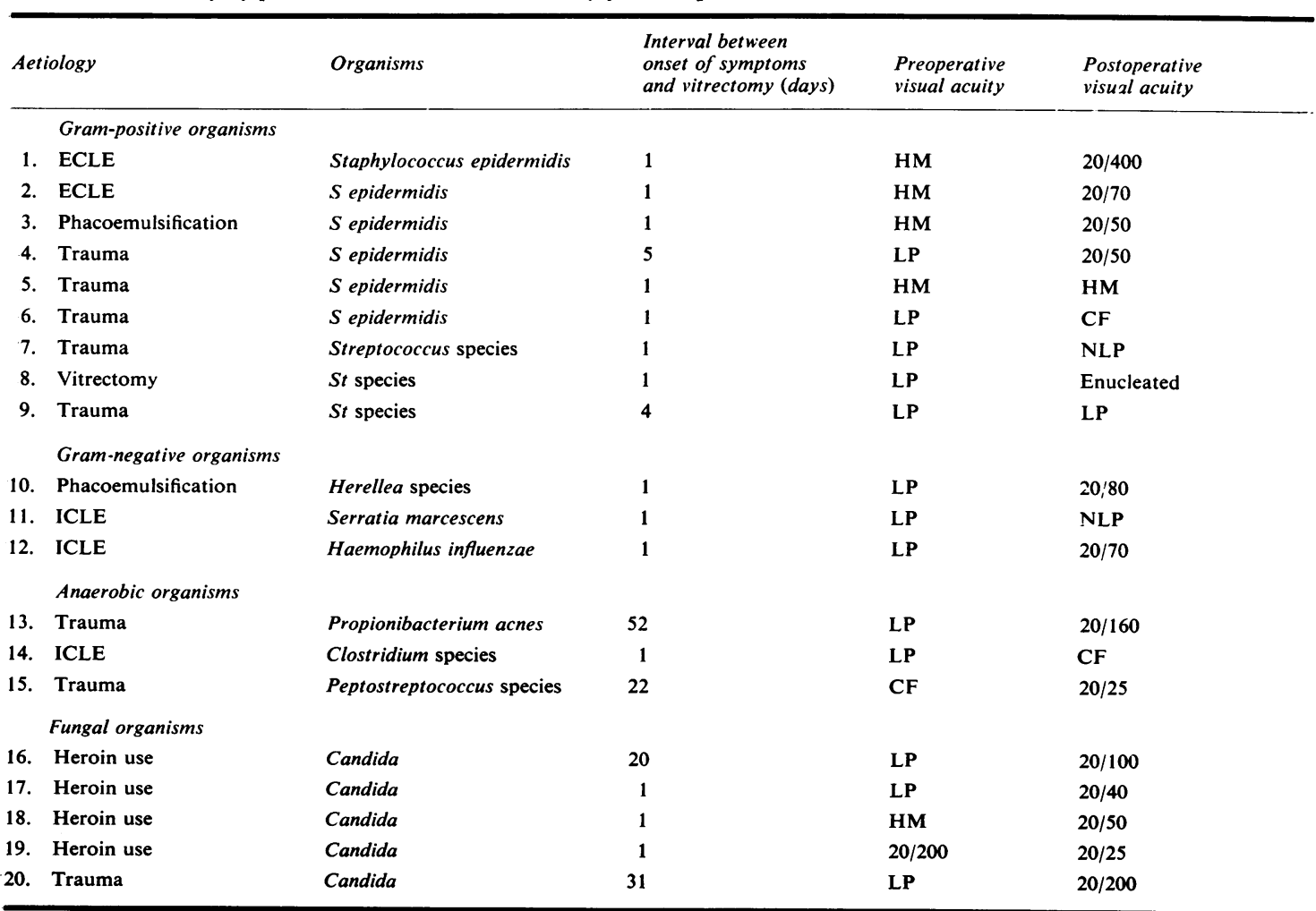

ECLE indicates extracapsular lens extraction; ICLE, intracapsular lens extraction; HM, hand motions; LP light perception; CF counting fingers: and NLP, no light perception. 
Table 3 Visual acuity after vitrectomy

\begin{tabular}{lcc}
\hline Visual acuity & No. of patients & $\%$ \\
\hline $20 / 100$ to $20 / 25$ & 10 & 50 \\
$20 / 400$ to $<20 / 100$ & 3 & 15 \\
Light perception to $<20 / 400$ & 4 & 20 \\
No light perception & 3 & 15 \\
\hline
\end{tabular}

$20 / 400$ to $20 / 100$ (Table 3 ). $15 \%$ had light perception to counting fingers visual acuity. One eye was enucleated and 2 eyes had no light perception. These 2 patients refused enucleation.

\section{Discussion}

Intravitreal antibiotic administration and vitrectomy in the management of endophthalmitis are rapidly gaining wide acceptance. ${ }^{2}$ Improved techniques of diagnosis have helped to differentiate bacterial and fungal endophthalmitis from other situations that mimic endophthalmitis, such as iridocyclitis and phacogenic uveitis. ${ }^{10}$ Conventional methods for the treatment of culture-proved endophthalmitis have not been very successful. ${ }^{11-13}$ In Allen and Mangiaracine's first series ${ }^{11}$ out of 22 cases of endophthalmitis only 4 retained useful vision. In their second report ${ }^{12}$ out of 9 eyes 4 regained useful vision. In Forster's series ${ }^{10}$ out of 14 culture-proved endophthalmitis cases only 3 retained useful vision.

These poor results are explained by the relative impermeability of the blood-retinal barrier to the antibiotics, even in the presence of endophthalmitis. ${ }^{14-16}$ Von Sallman et al. ${ }^{17}$ and Leopold ${ }^{18}$ were the first to study the intravitreal use of antibiotics in the treatment of experimental endophthalmitis in rabbits. However, the use of intravitreal antibiotics was abandoned for unknown reasons.

In 1974 we reported 5 cases of culture-proved endophthalmitis that were treated by intravitreal injection. ${ }^{19}$ Since then many other reports have been published. ${ }^{210}$

We studied the role of vitrectomy in the management of experimental endophthalmitis and the maximum dose of antibiotics that could be safely added to vitrectomy infusion fluids. ${ }^{3}$ In experimental studies eyes treated with intravitreal antibiotics alone and those treated with vitrectomy and antibiotics recovered. However, the recovery was much faster and the ocular media were clearer in the eyes treated by vitrectomy and antibiotics than in those receiving intravitreal antibiotics. ${ }^{45}$

In the present series $65 \%$ of the eyes recovered visual acuity of $20 / 400$ or better. An analysis of our data has shown 2 factors influencing the final visual outcome: the duration between the onset of infection and institution of therapy, and the nature of the infecting organism. Eyes infected with a marked exotoxin-producing organism had the worse prognosis. Three eyes $(15 \%)$ were lost; each had an infection from an organism that was capable of marked exotoxin and proteolytic enzyme production. Results were better when therapy was initiated within 36 hours after the onset of infection, except for anaerobes and fungal infections, which progress slowly.

We recommend vitrectomy when the diagnosis of endophthalmitis is established. This is usually 24 hours after aqueous and vitreous tap and initial injection of intravitreal antibiotics have been performed. In all cases vitrectomy influsion fluid should contain a nontoxic dose of appropriate antibiotics. ${ }^{2}$ In bacterial and fungal endophthalmitis, when the vitreous is severely involved, we perform vitrectomy as the primary procedure. Because of the toxicity of amphotericin B we do not recommend adding this to the infusion fluid, but it should be injected intravitreally at the end of the procedure. Recently we have evaluated the toxicity of amphotericin B methyl ester. ${ }^{20}$ This compound can be added to vitrectomy infusion fluid in a dose of $10 \mu \mathrm{g} / \mathrm{ml}$.

This study was supported in part by training grant EY703802, by core grant 1P30EY01792 from the National Institutes of Health, Bethesda, Maryland, and by the Lions of Illinois Foundation, Chicago.

\section{References}

${ }^{1}$ Peyman GA, Sanders DR. Advances in Uveal Surgery, Vitreous Surgery, and the Treatment of Endophthalmitis. New York: Appleton-Century-Crofts, 1975.

${ }^{2}$ Peyman GA, Vastine D, Raichand M. Experimental aspects and their clinical applications. In Symposium: Postoperative endophthalmitis. Trans Am Acad Ophthalmol Otolaryngol 1978; 85: 374-85.

${ }^{3}$ Stainer GA, Peyman GA, Meisels H, et al. Toxicity of selected antibiotics in vitreous replacement fluids. Ann Ophthalmol 1977; 9: 615-8.

${ }^{4}$ McGetrick JJ, Peyman GA. Vitrectomy in experimental endophthalmitis: II. Bacterial endophthalmitis. Ophthalmic Surg 1979; 10: 87-92.

${ }^{5}$ Huang K, Peyman GA, McGetrick JJ. Vitrectomy in experimental endophthalmitis: I. Fungal endophthalmitis. Ophthalmic Surg 1979; 10: 84-6.

${ }^{6}$ Morgan B, Larson B, Peyman GA, West CS. Toxicity of antibiotic combination for vitrectomy infusion fluid. Ophthalmic Surg 1979; 10: 74-7.

'Cazeau T, Mason GI, Peyman GA. Effects of vitrectomy infusion solutions containing oxacillin, methicillin or lincomycin. Ann Ophthalmol 1979; 8: 1247-51.

${ }^{8}$ Axelrod AJ, Peyman GA. Intravitreal amphotericin B of experimental fungal endophthalmitis. Am J Ophthalmol 1973; 76: 584-8.

${ }^{9}$ Axelrod AJ, Peyman GA, Apple DJ. Toxicity of intra- 
vitreal infection of amphotericin B. Am $J$ Ophthalmol 1973; 76: 578-83.

${ }^{10}$ Forster RK, Zachary IG, Cottingham AJ Jr, Norton EWD. Further observations on the diagnosis, cause, and treatment of endophthalmitis. Am J Ophthalmol 1976; 81: $52-6$.

${ }^{11}$ Allen HG, Mangiaracine AB. Bacterial endophthalmitis after cataract extraction: I. A study of 22 infections in 20000 operations. Arch Ophthalmol 1964; 72: 454-62.

${ }^{12}$ Allen HG, Mangiaracine AB. Bacterial endophthalmitis after cataract extraction: II. Incidence of 32000 consecutive operations with special reference to preoperative topical antibiotics. Arch Ophthalmol 1974; 91: 3-7.

${ }^{13}$ Forster RK. Endophthalmitis: diagnostic cultures and visual results. Arch Ophthalmol 1972; 92: 387-92.

${ }^{14}$ Peyman GA, Bok D. Peroxidase diffusion in the normal and laser coagulated primate retina. Invest Ophthalmol 1972; 11: $35-45$.

${ }^{15}$ Kasbeer RT, Peyman GA, May D, Homer PI. Penetration of amikacin into the aphakic eye. Albrecht von Graefes Arch Klin Ophthalmol 1975; 196: 85-94.

${ }^{16}$ Peyman GA, May DR, Homer PI, Kasbeer RT. Penetration of gentamycin into the aphakic eye. Ann Ophthalmol 1977; 7: 871-80.

${ }^{17}$ von Sallman L, Meyer K, DiGrandi J. Experimental study on penicillin treatment of ectogenous infection of vitreous. Arch Ophthalmol 1944, 32: 179-89.

${ }^{18}$ Leopold IH. Intravitreal penetration of penicillin and penicillin therapy of infections of the vitreous. Arch Ophthalmol 1945; 33: 211-6.

${ }^{19}$ Peyman GA, Vastine D, Crouch ER, Herbst RW. Clinical use of intravitreal antibiotics to treat bacterial endophthalmitis. Trans Am Acad Ophthalmol Otolaryngol 1974; 78: OP 862-75.

${ }^{20}$ Raichand M, Peyman GA, West CS, Hammond G, Zweig K. Amphotericin B methyl ester in the treatment of experimental fungal endophthalmitis: toxicity and efficacy of vitrectomy fluids. Ophthalmic Surg in press. 